\title{
APPROPRIATE EMPIRICAL MANAGEMENT OF MICROBIAL INFECTIONS IN A TERTIARY CARE HOSPITAL: A COST- EFFECTIVENESS APPROACH
}

\author{
ONCHARI DIVINAH N, JOSIN MARY SIMON*, SNEHA TOMY, ARUN PRASATH.R, SIVAKUMAR V
}

Department of Pharmacy Practice, PSG College of Pharmacy, Peelamedu, Coimbatore, Tamil Nadu, India. Email: josinmary58@gmail.com

Received: 07 September 2017, Revised and Accepted: 30 October 2017

ABSTRACT

Objective: Antibiotics are mostly prescribed empirically to decrease health-care costs. This has led to the misuse of antibiotics thereby making them inefficient in the treatment of infections. The aim of this study was to determine the appropriate, cost-effective drug for the empirical therapy in microbial infections.

Methods: A prospective observational study was conducted for a period of 6 months. Cost-effectiveness ratio (CER) of these antibiotics prescribed was calculated to determine the cost-effective drugs for the common microorganisms and common infections.

Results: In a population of 205 patients, 54.6\% were treated based on antibiotic sensitivity pattern whereas $45.3 \%$ were treated empirically. In known microbial infections, the prevailing microorganism was extended-spectrum beta-lactamase (ESBL) producing Escherichia-coli (14.3\%), Staphylococcus aureus (10.6\%), Pseudomonas aeruginosa (9.8\%), Klebsiella pneumonia (9.8\%), and K. pneumoniae ESBL (6.81\%). Based on the CER, the most cost-effective drugs for these organisms were found to be ciprofloxacin, clindamycin, ofloxacin, levofloxacin, and amikacin, respectively. In unknown microbial infection (empirical treatment), Diabetic Foot Infection (DFT) (25.8\%), respiratory tract infection (RTI) (23.6\%), and urinary tract infection (UTI) (16.1\%) were the most common infections. The most cost-effective drugs for these infections were clindamycin, levofloxacin, and azithromycin, respectively. The predominant microorganism in DFT was found to be S. aureus (71\%), in UTI was found to be E. coli ESBL (52\%), and in RTI were found to be P. aeruginosa (42.4\%) and K. pneumonia (32.3\%).

Conclusion: Appropriate empirical antibiotic treatment is associated with a lower medical cost and a better success rate in patients with microbial infections.

Keywords: Antibiotics, Cost-effectiveness analysis, Empirical therapy, Infection, Microorganisms, Sensitivity pattern.

(C) 2018 The Authors. Published by Innovare Academic Sciences Pvt Ltd. This is an open access article under the CC BY license (http://creativecommons. org/licenses/by/4. 0/) DOI: http://dx.doi.org/10.22159/ajpcr.2018.v11i2.22441

\section{INTRODUCTION}

Infection is the invasion and multiplication of disease-causing microbes into a human's body tissue and the reaction of the host tissue to these microbes and the toxins they produce. Infection is a common occurrence that has required patients to seek medical care and for antibiotics to be prescribed [1]. Antibiotics have made a significant contribution to improving the health of patients with microbial infections [2]. The unnecessary prescribing of antibiotics have led to increased cost of care by increasing resistance, drug cost, and cost for laboratory test [3]. Hence, while prescribing the antibiotic, important considerations like the accurate diagnosis of infection, understanding the difference between empirical and definitive therapy, cost-effective oral agents for the shortest duration should be included.

Antibiotic resistance is a major worldwide problem, and its implication on public health is becoming overwhelming [4-6]. Culture test helps to identify the causative organism and the effective drugs against these organisms can be determined by susceptibility tests. Cost is one among the various factors to be considered [7]. Hence, the antibiotics are mostly prescribed empirically to decrease the health-care cost.

A vast majority of infections are treated empirically, therefore, the choice of an antimicrobial agent should be adjusted by local sensitivity pattern as the rising prevalence of multidrug- resistant infections cause more complexity in the empirical treatment of these infections. Awareness of local antimicrobial resistance trends among microbial isolates is important for the appropriate use of antibiotics and for the evidence-based recommendations in the empirical antibiotic treatment of infections [8]. Therefore, pharmacoeconomic decision analysis is a way to convey the relative value of beneficial treatments, and the choice must be directed to obtain maximum benefit to the society. The alternative with the lowest cost to effectiveness ratio (CER) is preferred for the microbial infections. This study determines the appropriate, cost-effective antibiotics for the empirical therapy for the infections based on the sensitivity pattern of prevailing microorganisms. There is need to improve our antibiotic prescribing practices and to strengthen research to identify cost-effective strategies for controlling resistance. This could ameliorate the care of the individual patient tremendously [9].

\section{METHODS}

A prospective observational study was conducted over a period of 6 months from March 2016 to August 2016 in a multispecialty tertiary care hospital in Coimbatore, India. The study was approved to be conducted in PSG Institute of Medical Science and Research by the Institutional Human Ethics Committee (IHEC) (ref no: 16/092 dated on $14 / 03 / 2016)$. Patients receiving only one antibiotic who were treated either empirically or with culture sensitivity tests were included in the study. During this period 205 patients were enrolled in the study based on inclusion and exclusion criteria. Non-hospitalized, patients with incomplete and insufficient data/record and who received antibiotic as prophylaxis were excluded in the study. Data were collected using data collection form from the sources; laboratory tests and patient case file. The patients enrolled in the study were reviewed for 2 weeks. The total cost of each antibiotic was calculated based on the unit cost of drug, frequency and the number of days of administration.

Total cost $=$ Unit cost $\times$ frequency $\times$ number of days 
Study subjects were grouped based on the culture test; known microorganisms (sensitivity pattern therapy group) and unknown microorganisms (empirical therapy group). The cost-effectiveness analysis by CER was performed for common antibiotics used in both groups.

$$
\mathrm{CER}=\frac{\text { Total cost of antibiotic (INR) }}{\text { Outcome }(\text { success rate } \%)}
$$

The outcome was calculated as the percentage of successful treatments. CER was calculated for antibiotics prescribed in both the groups. From this analysis, the drug with the least CER was the most cost-effective drug of choice.

\section{RESULTS}

During the study period, 205 subjects met the criteria. Out of these 205 patients, $66.83 \%$ were male patients, and $33.17 \%$ were female patients. $40 \%$ of these patients were hospitalized for 6-10 days.

The study population was divided into two groups based on the culture test: Culture test with known microorganisms (sensitivity pattern therapy group) and culture test with the unknown microorganism (empirical therapy group). Table 1 summarizes the antibiotics mostly prescribed for the common microorganisms and infections.

Cost-effectiveness analysis of antibiotics for most commonly known organisms and unknown microorganisms were done, and a decision tree was prepared based on CER. In Escherichia coli extended-spectrum beta-lactamase (ESBL) infection, the most common cost-effective drug was found to be amikacin (CER=29.03) followed by ceftriaxone (CER=44.92) as shown in Table 2 .

Likewise, the cost-effectiveness analysis was performed for the other known microorganism as reported in Table 3.

Similarly, a cost-effectiveness analysis was performed for microbial infection with unknown microorganism and reported in Table 4.

The predominant microorganism in diabetic foot infection (DFT) was found to be Staphylococcus aureus (71\%), E. coli ESBL (52\%) in urinary tract infection (UTI) then Pseudomonas aeruginosa (42.4\%), and Klebsiella pneumonia $(32.3 \%)$ in respiratory tract infection (RTI)

Comparison of antibiotics of preference suggested in both known and unknown microbial infections showed that clindamycin was the best drug of choice for DFI and infections caused by $S$. aureus. It is also evident from the study that levofloxacin is the best drug of choice for RTI and infections caused by K. pneumonia, which is one of the common causative organisms for RTI. P. aeruginosa was also a predominant isolate in RTI, and the best drug of choice was ciprofloxacin. In UTI, the most common causative agent was E. coli ESBL, and nitrofurantoin was found to be the best alternative Table 5 represents the above data.

\section{DISCUSSION}

In this study, demographic characteristics of the participants involved in the study were categorized based on gender and age distribution. A study conducted by Thomas et al. in South India indicated the majority of the patients were male $68.5 \%$, the age distribution of these patients was between 41 and 60 years (43.6\%). Their study also showed that cephalosporins $(73.0 \%)$ are the most prescribed class of antibiotics followed by fluoroquinolones (53.9\%) which are in accordance with our study [10]. As per the study conducted by Dominick et al., it was reported that amikacin, piperacillin+tazobactam, cefepime, and nitrofurantoin were the most preferred drug in E. coli and K. pneumonia ESBL infection to avoid resistance to beta-lactams (meropenem and imipenem) which were expensive and could be used for more severe infections instead [11]. As stated by Rayner and Munckhof in their study conducted in Poland, penicillinase-resistant penicillins (flucloxacillin and dicloxacillin) were the best drugs in the treatment of severe infections of methicillin-sensitive S. aureus and acute infections, the first generation cephalosporins, and clindamycin could be used [4].

A study conducted by Suleiman et al. revealed Ciprofloxacin to be the most cost-effective treatment for sensitive isolates of $P$. aeruginosa when compared to ceftazidime and gentamicin. In resistant isolates, amikacin was dominantly more cost-effective when compared to meropenem and imipenem [6]. As per Canadian study conducted by Chow et al., showed clindamycin dominated other drugs such as cephalexin, cloxacillin, levofloxacin, and amoxicillin+clavulanate with the lowest CER in the treatment of DFT [12]. Radji et al. conducted a study in Indonesia found that the most common infecting microorganisms on pus culture to be

Table 1: Antibiotics mostly prescribed for the common microorganisms and infections

\begin{tabular}{|c|c|c|}
\hline Groups & Organisms/infections (\%) & Antibiotics prescribed (\%) \\
\hline \multirow[t]{14}{*}{ Sensitivity pattern therapy group (known microorganisms) } & E. coli ESBL (14.39) & Amikacin (22) \\
\hline & & Ceftriaxone (17) \\
\hline & & Cefoperazone+sulbactam (17) \\
\hline & S. aureus (10.6) & Amoxicillin+clavulanate (59) \\
\hline & & Piperacillin+tazobatam (24) \\
\hline & & Clindamycin (19) \\
\hline & P. aeruginosa (9.84) & Piperacillin+tazobatam (36) \\
\hline & & Ofloxacin (23) \\
\hline & K. pneumoniae (9.84) & Levofloxacin (31) \\
\hline & & Ceftriaxone (31) \\
\hline & & Piperacillin-tazobactam (23) \\
\hline & K. pneumoniae ESBL (6.81) & Piperacillin+tazobactam (36) \\
\hline & & Meropenem (22) \\
\hline & & Amikacin (21) \\
\hline \multirow[t]{6}{*}{ Empirical therapy group (infections) } & DFI (25.80) & Amoxicillin+clavulanate (44.18) \\
\hline & RTI (23.65) & Ceftriaxone (41.17) \\
\hline & & Azithromycin (17.64) \\
\hline & & Levofloxacin (14.75) \\
\hline & UTI (16.12) & Cefoperazone+sulbactam (21.05) \\
\hline & & Piperacillin+tazobactam (20.1) \\
\hline
\end{tabular}

E-coli: Escherichia coli, ESBL: Extended-spectrum beta-lactamase, S. aureus: Staphylococcus aureus, P. aeruginosa: Pseudomonas aeruginosa, K. pneumonia: Klebsiella pneumonia, DFI: Diabetic foot infection, RTI: Respiratory tract infection, UTI: Urinary tract infection 
Table 2: CEA of Antibiotics in E. coli ESBL infection

\begin{tabular}{lllll}
\hline Antibiotic & Total cost (INR) & Mean cost per patient (INR) & Success (\%) & CER \\
\hline Amikacin & 2257.95 & 250.88 & 77.7 & 29.03 \\
Ceftriaxone & 3850.80 & 550.11 & 85.7 & 44.92 \\
Cefoperazone+sulbactam & 41445.62 & 5920.8 & 100 & 414.45 \\
\hline
\end{tabular}

CER: Cost-effectiveness ratio

Table 3: Choice of antibiotic in common organisms based on CER

\begin{tabular}{llll}
\hline Microorganism & Antibiotic of first preference & CER & Antibiotic of $\mathbf{2}^{\text {nd }}$ preference \\
\hline E-coli ESBL & Amikacin & 29.03 & Ceftriaxone \\
Pseudomonas aeruginosa & Ofloxacin & 24.4 & Clindamycin \\
Klebsiella pneumoniae & Levofloxacin & 3.19 & Ceftriaxone \\
Klebsiella pneumoniae ESBL & Amikacin & 16.09 & Amoxicillin+clavulanate \\
Staphylococcus aureus & Clindamycin & 145.6 & Amoxicillin+clavulanate \\
\hline
\end{tabular}

E. coli: Escherichia coli, ESBL: Extended-spectrum beta-lactamase, S. aureus: Staphylococcus aureus, P. aeruginosa: Pseudomonas aeruginosa, K. pneumonia: Klebsiella pneumonia, CER: Cost-effectiveness ratio

Table 4: Choice of antibiotics in common infections based on CER

\begin{tabular}{lllll}
\hline Microbial infection & Antibiotic of first preference & CER & Antibiotic of second preference & CER \\
\hline UTI & Azithromycin & 3.15 & Nitrofurantoin & 4.71 \\
DTI & Clindamycin & 208.25 & Cefoperazone+sulbactam & 315.77 \\
RTI & Levofloxacin & 3.47 & Cefpodoxime & 6.15 \\
\hline
\end{tabular}

CER: Cost-effectiveness ratio, DFI: Diabetic foot infection, RTI: Respiratory tract infection, UTI: Urinary tract infection

Table 5: Comparison of antibiotics of preferences for both groups

\begin{tabular}{lll}
\hline Infection & Organism & Drug of choice \\
\hline DTI & Staphylococcus aureus & Clindamycin \\
RTI & Klebsiella pneumoniae & Levofloxacin \\
& Pseudomonas aeruginosa & Ciprofloxacin \\
UTI & E-coli ESBL & Nitrofurantoin \\
\hline
\end{tabular}

DFI: Diabetic foot infection, RTI: Respiratory tract infection, UTI: Urinary tract infection, E-coli: Escherichia coli, ESBL: Extended-spectrum beta-lactamase, S. aureus: Staphylococcus aureus, P. aeruginosa: Pseudomonas aeruginosa,

K. pneumonia: Klebsiella pneumonia

Staphylococcus spp. $47.5 \%$, Pseudomonas spp. $16.9 \%$, and E. coli $10.2 \%$, Streptococcus spp. $8.5 \%$. Overall, $37.2 \%$ of DFI was caused by a single organism, and $62.8 \%$ had polymicrobial infections [13]. Commonly administered antibiotics were ceftriaxone $40.0 \%$, ciprofloxacin $11.4 \%$, and meropenem $8.6 \%$. The empiric antibiotic regimen of clindamycin and ciprofloxacin covered $85 \%$ of $S$. aureus and $78 \%$ of Gram-negative species of DFI in a study conducted by de Vries et al. in Netherlands [14].

An Egyptian study by Harwan et al. reported that respiratory fluoroquinolone such as Levofloxacin should be regarded as a first line antimicrobials in admitted and outpatients due to their association with increased clinical response, faster resolutions, greater improvement of signs and symptoms and shorter hospital length of stay [15]. According to a study conducted in Nigeria by Egbe et al., Klebsiella pneumoniae was reported to be the predominant isolate fluoroquinolones, beta-lactams, and Gentamicin showed moderate to high activity [16]. Varotto, Maria found the four most frequent isolates in RTI to be P. aeruginosa $24 \%$, Streptococcus pyogenes 18\%, Staphylococcus spp. 17\%, and Klebsiella spp. $8 \%$.

IDSA guidelines state that the first line treatment for UTI is nitrofurantoin and fluoroquinolones. Dudas et al. reported that $80 \%$ of UTI was caused by E. coli in Northern California. Most patients were treated empirically using beta-lactams, nitrofurantoin, fosfomycin tromethamine, fluoroquinolones, and trimethoprim eradicate Gramnegative flora, hence, provide best long-term cures [17]. In the USA, Bouza et al. found E. coli to be the main etiological agent of UTI [18].
A study in Turkey on the evaluation of empirical treatment by Yüksel et al. reported that the most common causative agent was E. coli $87 \%$, Klebsiella $10 \%$. Nitrofurantoin was the most active agent against $E$. coli followed by amikacin and ceftriaxone [19].

This study had some limitations. First, it was conducted for a short period ( 6 months), therefore, the sensitivity pattern could not be thoroughly assessed. Second, the cost focused on only antibiotics; other medical expenses were not included. Finally, the data were analyzed from one of the tertiary care hospitals in Southern India.

\section{CONCLUSION}

Appropriate empirical antibiotic treatment is associated with a lower medical cost and a better success rate in patients with microbial infections. Efforts to improve the appropriateness of empirical antibiotic treatment should be dictated by the benefit inherent inappropriate treatment. The choice of antimicrobial agent should be adjusted by local sensitivity pattern as there might be geographic differences in sensitivity patterns. In addition, prudent use of available antibiotic drugs, improved methods of prediction, and rapid detection of resistance is clearly needed.

\section{REFERENCES}

1. Little P, Watson L, Morgan S, Williamson I. Antibiotic prescribing and admissions with major suppurative complications of respiratory tract infections: A data linkage study. Br J Gen Pract 2002;52:187-90.

2. Feldman C. Appropriate management of lower respiratory tract infections in primary care. Prim Care Respir J 2004;13:159-66.

3. Harbarth S, Samore MH, Lichtenberg D, Carmeli Y. Prolonged antibiotic prophylaxis after cardiovascular surgery and its effect on surgical site infections and antimicrobial resistance. Circulation 2000;101:2916-21.

4. Chow I, Lemos EV, Marr P, Machado M, Einarson TR. Pharmacoeconomic analysis of guidelines for treating mild diabetic foot infections: A decision-tree model for Canada. Can J Hosp Pharm 2008;61:412-21.

5. National Institute of Allergy and Infectious disease (NIAID). National Institute of Allergy and Infectious Diseases; 2013. Available from: http://www.niaid,nih.gov/topics/antimicrobialresistance/Pages/default. aspx.

6. Suleiman I, Bamiro SB, Mendie UE. Cost-effectiveness of six drugs for the treatment of Pseudomonas aeruginosa infections in Nigeria. Int $\mathrm{J}$ 
Pharm Pharm Sci 2014;6:239-44.

7. Kerr JR, Barr JG, Smyth ET, O'Hare J, Bell PM, Callender ME, et al. Antibiotic pharmacoeconomics: An attempt to find the real cost of hospital antibiotic prescribing. Ulster Med J 1993;62:50-7.

8. Sedighi I, Solgi A, Amanati A, Alikhani MY. Choosing the correct empirical antibiotic for urinary tract infection in pediatric: Surveillance of antimicrobial susceptibility pattern of Escherichia coli by E-test method. Iran J Microbiol 2014;6:387-91.

9. Thomas B, Mathew L, Jose J, Rathinavelu M. Assessment of antibiotic sensitivity pattern of microorganisms and their cost-effectiveness in private cooperative hospital. Asian J Pharm Clin Res 2004;7:155-9.

10. Salvatore DJ, Beth H. Resman-targoff. treatment options for urinary tract infections caused by extended-spectrum B-lactamase-producing Escherichia coli and Klebsiella pneumoniae. Am J Hosp Pharm 2015;7:53-6.

11. Rayner C, Munckhof WJ. Antibiotics currently used in the treatment of infections caused by Staphylococcus aureus. Int Med J 2005;3:3-16.

12. Radji M, Putri CS, Fauziyah S. Antibiotic therapy for diabetic foot infections in a tertiary care hospital in Jakarta, Indonesia. Europe PMC 2014:8:221-4

13. de Vries MG, Ekkelenkamp MB, Peters EJ. Are clindamycin and ciprofloxacin appropriate for the empirical treatment of diabetic foot infections? Eur J Clin Microbiol Infect Dis 2014;33:453-6.

14. Harwan WA, Abbassi MM, May M. Pharmacoeconomic study of antibiotics used in the treatment of lower respiratory tract infections in ICU patients: A case study in an Egyptian hospital. Bull Fac Pharm Cairo Univ 2014;5:135-44.

15. Egbe CA, Ndiokwere C, Omoregie R. Microbiology of lower respiratory tract infections in Benin City, Nigeria. Malays J Med Sci 2011;18:27-31

16. Dudas V, Sarah B, Trivedi KT. Implementation of an antimicrobial stewardship program targeting resistance with UTI in three community long-term care facilities: A quasi-experimental study using time-series analysis. Antimicrob Resist Infect Control 2015;4:2-8.

17. Bouza JM, Sangrador C, Mendez CP. The etiology of urinary tract infections and the antimicrobial susceptibility of urinary pathogens. Rev Esp Quimioter 2005;8:124-35.

18. Yüksel S, Oztürk B, Kavaz A. Antibiotic resistance of urinary tract pathogens and evaluation of empirical treatment in Turkish children with urinary tract infections. Int J Antimicrob Agents 2006;28:413-6.

19. Bisht R, Aatiyar A, Singh R, Mittal P. Antibiotic resistance - A global issue of concern. Asian J Pharm Clin Res 2009;2:34-7. 